\title{
ON AUTOMORPHISMS OF L.C. GROUPS
}

\author{
JUSTIN PETERS
}

\begin{abstract}
The left regular representation $\lambda$ of a locally compact group $G$ generates a $W^{*}$-algebra $\Re(\lambda)$, and each topological automorphism $\tilde{\alpha}$ of $G$ has a natural extension to an automorphism $\tilde{\alpha}$ of $\mathscr{R}(\lambda)$. It is proved that an automorphism $\beta$ of $\Re(\lambda)$ is of the form $\beta=\tilde{\alpha}$ for $\alpha \in \operatorname{Aut}(G)$ iff $\beta$ leaves a certain cone in $\mathscr{R}(\lambda)$ invariant.
\end{abstract}

Recently a number of important results concerning automorphisms of $W^{*}$-algebras have been obtained (see e.g. [2], [4]), and in some cases these results have analogues in automorphisms of locally compact groups, or can be applied directly to yield facts about group automorphisms [6]. Let $\lambda$ denote the left regular representation of a locally compact group $G(\lambda(x), x \in G$, operates on an $L^{2}(G)$-function by left translation by $\left.x^{-1}\right)$ and $(R(G)$ be the double commutant of $\lambda(G)=\{\lambda(x): x \in G\}$, or the $W^{*}$-algebra generated by $\lambda(G)$. There is a natural imbedding of $\operatorname{Aut}(G) \hookrightarrow$ Aut ${ }^{\prime}(G)$ : to $\alpha \in$ Aut $(G)$ there corresponds a unique $\tilde{\alpha} \in$ Aut $\mathscr{R}(G)$ satisfying $\tilde{\alpha} \lambda(x)=$ $\lambda(\alpha(x))$. In this note we characterize those automorphisms of $\mathscr{R}(G)$ which come from automorphisms of $G$ (via the imbedding) as the set of automorphisms of $\Omega(G)$ which leave a certain cone fixed. Also, a connection between automorphisms of $\Re(G)$ and the measure algebra $\mathscr{R}(G)$ is mentioned.

If $A$ is a Banach *-algebra, we denote by $A^{\prime+}$ the positive cone in the dual $A^{\prime}$ given by $\left\{f \in A^{\prime}: f\left(a^{*} a\right) \geqslant 0, a \in A\right\}$. There is also a cone $A^{+}=\{a \in$ $\left.A: f(a) \geqslant 0, f \in A^{\prime+}\right\}$ in $A$. A ray in $A^{\prime+}$ is a set of the form $R^{+} f, f \in A^{\prime+}$ $\left(R^{+}=\{r \in R: r \geqslant 0\}\right)$. A linear functional $f \in A^{\prime+}$ is said to lie on an extreme ray if $f=g+h, g, h \in A^{\prime+}$, implies $g, h \in R^{+} f$. The following proposition is an extension of a well-known theorem of Kelley and Vaught $[5]$.

Proposition. Let $A$ be a commutative Banach *-algebra with continuous involution, and $B \subset A$ a dense subalgebra. Suppose $\left\{e_{i}\right\} \subset B \cap A^{+}$is a (not necessarily bounded) approximate identity for $B$. Let $f \in A^{\prime+}$; then $f$ lies on an extreme ray if and only if $\theta f$ is multiplicative for some $\theta, 0 \neq \theta \in R^{+}$.

Proof. Let $f \in A^{\prime+}$ lie on an extreme ray and choose $b \in B$ such that

Received by the editors January 26, 1977.

AMS (MOS) subject classifications (1970). Primary 22D45.

Key words and phrases. Automorphism, $W^{*}$-algebra, Fourier algebra, measure algebra, group character. 
$\left\|b^{*} b\right\|<1$ and $f\left(b^{*} b\right)>0$. Define $g \in A^{\prime+}$ by $g(y)=f\left(y b^{*} b\right)$. Clearly $g$ is positive since $g\left(y^{*} y\right)=f\left((y b)^{*}(y b)\right) \geqslant 0$. Likewise $(f-g) \in A^{\prime+}$, for $(f-$ $g)\left(y^{*} y\right)=f\left(z^{*} z\right)$, where $z=\sum_{n=0}^{\infty}\left({ }_{n}^{1 / 2}\right) y\left(-b^{*} b\right)^{n}$. (Observe that if we adjoined an identity to $A$ in the canonical way we could write

$$
\left.z=y \sqrt{1-b^{*} b}=y \sum_{n=0}^{\infty}\left(\begin{array}{c}
1 / 2 \\
n
\end{array}\right)\left(-b^{*} b\right)^{n} .\right)
$$

Since we can write $f=g+(f-g)$ with $g,(f-g) \in A^{\prime+}$, it follows from the assumption on $f$ that $g=\mu f$, for some $\mu, 0 \leqslant \mu<\infty$. But $g\left(e_{i}\right)=$ $f\left(e_{i} b^{*} b\right) \neq 0$ for sufficiently large $i$, so $\mu>0$. This implies $\lim f\left(e_{i}\right)$ exists, and, in fact,

$$
\lim f\left(e_{i}\right)=\mu^{-1} \lim g\left(e_{i}\right)=\mu^{-1} \lim f\left(e_{i} b^{*} b\right)=\mu^{-1} f\left(b^{*} b\right)<\infty .
$$

Let

$$
\theta=\mu / f\left(b^{*} b\right)=1 / \lim f\left(e_{i}\right) .
$$

Note that $\theta$ does not depend on $b$. Then $\theta g=\mu \theta f$ and $\mu=\theta f\left(b^{*} b\right)$. Thus

$$
\theta g(y)=\theta f\left(b^{*} b\right) \theta f(y), \quad y \in A,
$$

or

$$
\theta f\left(b^{*} b y\right)=\theta f\left(b^{*} b\right) \theta f(y), \quad y \in A .
$$

Observe now that this last equation is valid if in place of $b^{*} b$ we have $a^{*} a$, $a \in A$, where $f\left(a^{*} a\right)=0$. For in that case $\left|f\left(a^{*} a y\right)\right|^{2} \leqslant f\left(a^{*} a\right) f\left((a y)^{*}(a y)\right)$ $=0$. Since any $x \in B^{2}$ can be written as a linear combination of elements of the form $b^{*} b,\left\|b^{*} b\right\|<1$, we have $\theta f(x y)=\theta f(x) \theta f(y), x \in B^{2}, y \in A$. Finally, using that $B^{2} \subset A$ is dense along with the continuity of $f$, we have that $\theta f(x y)=\theta f(x) \theta f(y)$ holds for all $x, y \in A$.

The converse is easy. For let $f \in A^{\prime+}$ be multiplicative and suppose $f=g+h, g, h \in A^{\prime+}$. Let $N_{f}$ (resp., $N_{g}$ ) be the null space of $f$ (resp., $g$ ). If $x \in N_{f}$, then $f\left(x^{*} x\right)=f\left(x^{*}\right) f(x)=0$, hence $g\left(x^{*} x\right)=0$. But then $|g(x)|^{2}<$ $g\left(x^{*} x\right)=0$, so $N_{g} \supset N_{f}$. This means $g=\mu f$ for some $\mu, 0 \leqslant \mu<\infty$, and, consequently, $f$ lies on an extreme ray.

We apply the foregoing proposition by taking $A$ to be $A(G)$, the Fourier algebra of a locally compact group $G$. Recall that $A(G)$ is a Banach*- algebra of continuous functions on $G$ vanishing at infinity under pointwise multiplication with complex conjugation as involution. If $C_{00}(G)$ denotes the continuous functions with compact support on $G$, then $B=C_{00}(G) \cap A(G)$ is dense in $A(G)$. Given a compact set $k \subset G$ there is a function $a_{k} \in B$, $0 \leqslant a_{k} \leqslant 1, a_{k} \mid k=1[3,3.2]$. If for each compact set $k \subset G$ we choose such an $a_{k}$, order the $k$ 's by inclusion and set $e_{k}=a_{k}^{2}=a_{k}^{*} a_{k}$, then $\left\{e_{k}\right\} \subset B \cap$ $A^{+}$constitutes an (unbounded) approximate identity for $B$, so the hypotheses of the proposition are satisfied.

Now $A(G)^{\prime}=R(G)$, and $\Delta(A(G)$ ), the spectrum of $A(G)$, is identified with $G$, or, more properly, $\lambda(G)$ (acting by pointwise evaluation) [3, 3.34]. Let $P=A(G)^{\prime+}$. The cone $P$ is not to be confused with the cone of positive 
operators in $\mathscr{R}(G)$ : indeed, $\lambda(x)(x \in G)$ is clearly in $P$, but $\lambda(x)$ is not even hermitian if $x \neq x^{-1}$. Applying the above proposition, we find that the set extreme rays of $P$ is precisely $\left\{R^{+} \lambda(x): x \in G\right\}$.

By an automorphism of a $W^{*}$ algebra we mean, of course, a *automorphism, and this will automatically be norm-preserving.

COROllaRY. An automorphism $\beta$ of $\mathcal{R}(G)$ comes from an automorphism of $G$ if and only if $\beta P=P$.

Proof. $P$ has a metrizable topology and so is well capped [1, 30.19]; hence by the Choquet theory $P$ is the closed convex hull of its extreme rays. If $\beta=\tilde{\alpha}, \alpha \in \operatorname{Aut}(G), \beta$ maps the set of extreme rays of $P$ onto itself, thus $\beta P=P$. Suppose, conversely, that $\beta P=P$. If $\beta \lambda(x)=S+T, S, T \in P$, then $\lambda(x)=\beta^{-1} S+\beta^{-1} T$. Thus $\beta^{-1} S=\mu \lambda(x)$, some $0 \leqslant \mu<\infty$, or $S=$ $\mu \beta \lambda(x)$, which means $\beta \lambda(x)$ lies on an extreme ray. Since $\|\beta \lambda(x)\|=1$, we must have $\beta \lambda(x)=\lambda(y)$, for some $y \in G$. Setting $y=\alpha(x)$ we obtain a map $\alpha: G \rightarrow G$. An easy argument now shows $\alpha \in \operatorname{Aut}(G)$.

Instead of looking at the extreme rays of a certain cone in ${ }^{\prime}(G)$ we could, of course, consider the extreme points of the unit ball in $\mathscr{R}(G), \operatorname{Ext}\left(\Re(G)_{1}\right)$. But any $\beta \in$ Aut $R(G)$ must map $\operatorname{Ext}\left(\Re(G)_{1}\right)$ onto itself, so in place of $\Re(G)_{1}$ we might take $\mathscr{R}(G)_{1}$, where $\mathscr{R}(G)$ is the measure algebra. Now $A(G) \subset C_{0}(G)$, the continuous functions on $G$ vanishing at infinity, so we can view $\mathscr{R}(G)=C_{0}(G)^{\prime}$ as a subalgebra of $\Re(G)$. If $\mathscr{R}(G)$ is given its own norm (and not the norm it inherits from $\mathscr{R}(G)$ ), then $\operatorname{Ext}\left(\Re(G)_{1}\right)=$ $\left\{e^{i \theta} \delta_{x}: \theta \in R, x \in G\right\}, \delta_{x}$ being the point mass at $x$. Indeed, if $\mu \in \mathfrak{N}(G)_{1}$, $\|\mu\|=1$, is nonatomic, then there are nonzero $\mu_{1}, \mu_{2} \in \mathfrak{N}(G)$, $\operatorname{support}\left(\mu_{i}\right)$ $\subset \operatorname{support}(\mu), i=1,2$, satisfying $\operatorname{support}\left(\mu_{1}\right) \cap \operatorname{support}\left(\mu_{2}\right)$ is a $|\mu|$-null set, and $\mu=\mu_{1}+\mu_{2}$ with $\left\|\mu_{1}\right\|+\left\|\mu_{2}\right\|=\|\mu\|=1$. Setting $V_{i}=\left\|\mu_{i}\right\|^{-1} \mu_{i}, i=$ 1 , 2, we have $\mu=\left\|\mu_{1}\right\| V_{1}+\left\|\mu_{2}\right\| V_{2}$, so $\mu$ is not extreme. On the other hand, $e^{i \theta} \delta_{x} \in \operatorname{Ext}\left(\mathscr{R}(G)_{1}\right)_{1}$. In fact, viewing $\mathfrak{R}(G) \subset \Re(G), e^{i \theta} \delta_{x}$ belongs to the larger unit ball $\Re(G)_{1}$, and $e^{i \theta} \delta_{x} \in \operatorname{Ext}\left(\Re(G)_{1}\right)$ by [7, 1.6.4].

Proposition. $\beta \in$ Aut $R(G)$ restricts to an isometric automorphism of TR $(G)$ if and only if $\beta=\tilde{\alpha} \circ \tilde{\gamma}$, where $\beta \in \operatorname{Aut}(G)$ and $\gamma$ is a group character.

Proof. Consider first that a group character acts as a ${ }^{*}$-automorphism of the group algebra $L^{1}(G)$ : defining $(\gamma f)(x)=\gamma(x) f(x), f \in L^{1}(G)$, we have that $(\gamma f) *(\gamma g)=\gamma(f * g), f, g \in L^{1}(G)$, and $(\gamma f)^{*}=\gamma f^{*}$, where $f^{*}(x)=$ $\Delta(x)^{-1} f\left(x^{-1}\right)^{-}$is the involution in $L^{1}(G)$. It is clear that $\gamma$ extends to an isometric *-automorphism of the measure algebra; for $\mu \in \mathfrak{N}(G)$, we have $d(\gamma \mu)(x)=\gamma(x) d \mu(x)$. Let $\gamma$ act on $\mathcal{R}(G)$ by defining $(\tilde{\gamma} T) g=\gamma(T(\bar{\gamma} g))$, $T \in \mathcal{R}(G), g \in L^{2}(G)$, where $\gamma$ acts by pointwise multiplication on $L^{2}(G)$; i.e., $\tilde{\gamma} T=\gamma T \bar{\gamma}$. To see this define a *automorphism of $\mathcal{R}(G)$, first note for $T \in \mathcal{R}(G), g, h \in L^{2}(G)$ that

$$
\begin{aligned}
\left((\tilde{\gamma} T)^{*} g, h\right) & =(g, \tilde{\gamma} T h)=(g, \gamma T \bar{\gamma} h)=(\bar{\gamma} g, T \bar{\gamma} h) \\
& =\left(T^{*} \bar{\gamma} g, \bar{\gamma} h\right)=\left(\gamma T^{*} \bar{\gamma} g, h\right)=\left(\tilde{\gamma} T^{*} g, h\right) .
\end{aligned}
$$


To show $\tilde{\gamma}$ is multiplicative, observe, if $f \in L^{1}(G), \tilde{\gamma} \lambda(f)=\lambda(\gamma f)$. (We use the same notation for the left regular representation of $L^{\prime}(G)$, which acts by left convolution on $L^{2}(G)$, as we do for the left regular representation of $G$, since the former is just the Bochner-integrated form of the latter.) So for $f, g \in$ $L^{1}(G)$,

$$
\begin{aligned}
\tilde{\gamma}(\lambda(f) \lambda(g)) & =\tilde{\gamma} \lambda(f * g)=\lambda(\gamma(f * g)) \\
& =\lambda((\gamma f) *(\gamma g))=\lambda(\gamma f) \lambda(\gamma g)=\tilde{\gamma} \lambda(f) \tilde{\gamma} \lambda(g) .
\end{aligned}
$$

Then use that $\left\{\lambda(f): f \in L^{1}(G)\right\} \subset \Re(G)$ is strongly dense and the fact that multiplication in $\Re(G)$ is jointly strongly continuous on bounded subsets to see $\tilde{\gamma}$ is multiplicative on $\mathcal{R}(G)$.

Suppose now $\beta \in$ Aut $\mathscr{R}(G)$ restricts to an isometric automorphism of $\Re(G)$. Then $\beta$ maps the unit ball of $\mathscr{R}(G)$ onto itself, hence $\operatorname{Ext}\left(\mathscr{N}(G)_{1}\right)$ is mapped onto itself. Thus $\beta \delta_{x}=e^{i \theta} \delta_{\alpha(x)}$, for some $\theta \in R$, and $\alpha: G \rightarrow G$. If we set $|\beta| \delta_{x}=\delta_{\alpha(x)}$, it is clear that $|\beta|$ is multiplicative on $\operatorname{Ext}\left(\mathscr{N}(G)_{1}\right)$, hence $\alpha \in \operatorname{Aut}(G)$. Set $\beta \delta_{x}=\gamma(x) \delta_{\alpha(x)}$. A simple argument shows $\gamma$ is multiplicative, hence is a group character. Thus $\beta$ agrees with $\tilde{\alpha} \circ \tilde{\gamma}$ on $\lambda(G)$, and since $\lambda(G)$ generates $\mathscr{R}(G)$, we must have $\beta=\tilde{\alpha} \circ \tilde{\gamma}$.

\section{REFERENCES}

1. G. Choquet, Lectures on analysis, Vol. II, Benjamin, New York, 1969.

2. A. Connes, Almost periodic states and factors of type $\mathrm{III}_{1}$, J. Functional Analysis 16 (1974), 415-445.

3. P. Eymard, L'algèbre de Fourier d'un groupe localement compact, Bull. Soc. Math. France 92 (1964), 181-236.

4. U. Haagerup, The standard form of von Neumann algebras, Math. Scand. 37 (1975), 271-283.

5. J. Kelley and R. Vaught, The positive cone in Banach algebras, Trans. Amer. Math. Soc. 74 (1953), 44-55.

6. J. Peters and T. Sund, Automorphisms for locally compact groups, Univ. Oslo Inst. Math. Preprint Series, no. 14, 1976.

7. S. Sakai, $C^{*}$-algebras and $W^{*}$-algebras, Springer-Verlag, Berlin and New York, 1971.

8. M. Takesaki and N. Tatsuuma, Duality and subgroups, Ann. of Math. (2) 93 (1971), 344-364.

Department of Mathematics, Iowa State University, Ames, Iowa 50011 